\title{
Unsupervised delineation of the vessel tree in retinal fundus images
}

\author{
N. Strisciuglio \& M. Vento \\ Department of Computer Eng. and Electrical Eng. and Applied Mathematics \\ University of Salerno
}

G. Azzopardi

Department of Intelligent Computer Systems

University of Malta, Malta

N. Strisciuglio \& G. Azzopardi \& N. Petkov

Johann Bernoulli Institute for Mathematics and Computer Science

University of Groningen, the Netherlands

\begin{abstract}
Retinal imaging has gained particular popularity as it provides an opportunity to diagnose various medical pathologies in a non-invasive way. One of the basic and very important steps in the analysis of such images is the delineation of the vessel tree from the background. Such segmentation facilitates the investigation of the morphological characteristics of the vessel tree and the analysis of any lesions in the background, which are both indicators for various pathologies. We propose a novel method called $B$-COSFIRE for the delineation of the vessel tree. It is based on the classic COSFIRE approach, which is a trainable nonlinear filtering method. The responses of a $B$-COSFIRE filter is achieved by combining the responses of difference-of-Gaussians filters whose areas of support are determined in an automatic configuration step. We configure two types of $B$-COSFIRE filters, one that responds selectively along vessels and another that is selective to vessel endings. The segmentation of the vessel tree is achieved by summing up the response maps of both types of filters followed by thresholding. We demonstrate high effectiveness of the proposed approach by performing experiments on four public data sets, namely DRIVE, STARE, CHASE_DB1 and HRF. The delineation approach that we propose also has lower time complexity than existing methods.
\end{abstract}

Keywords: delineation, retinal image analysis, trainable filters, vessel segmentation.

\section{INTRODUCTION}

Retinal fundus imaging is a non-invasive technique that has gained popularity for the diagnosis of several pathologies, including diabetic retinopathy, glaucoma, hypertension, arteriosclerosis and multiple sclerosis. The automatic analysis of retinal images may be used to assist medical specialists to diagnose such pathologies.

The separation of the blood vessels from the rest of the image is an important segmentation step, which facilitates the subsequent analysis. For instance, the detection of vessel bifurcations, whose progression may provide signs for cardiovascular diseases, may be better detected in the segmented image. Moreover, subtracting the delineated vessel tree from the background provide better opportunity for the automatic detection of any retinal lesions.

There are two categories of methods that have been proposed so far, namely supervised (Staal et al. 2004, Soares et al. 2006, Ricci and Perfetti 2007,
Marin et al. 2011, Fraz et al. 2012) and unsupervised (Hoover et al. 2000, Jiang and Mojon 2003, Mendonca and Campilho 2006, Al-Rawi et al. 2007) approaches. Supervised approaches form pixel-wise feature vectors and learn a binary classifier that labels pixels as vessel or non-vessel. On the other hand, unsupervised approaches, typically rely on tracking, filtering or morphological operations followed by thresholding. Supervised methods require reasonably-sized data sets of training images and corresponding ground truth, which is usually very difficult and expensive to obtain.

We propose a novel unsupervised delineation algorithm that is based on the Combination of Receptive Fields (CORF) computational model of a simple cell in visual cortex (Azzopardi and Petkov 2012, Azzopardi et al. 2014) and its implementation called Combination of Shifted Filter Responses (COSFIRE) (Azzopardi and Petkov 2013). It uses two types of COSFIRE filters, one that gives a response along the vessels and the other one that selectively 

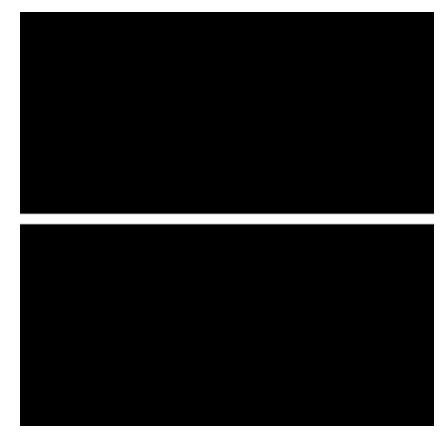

(a)

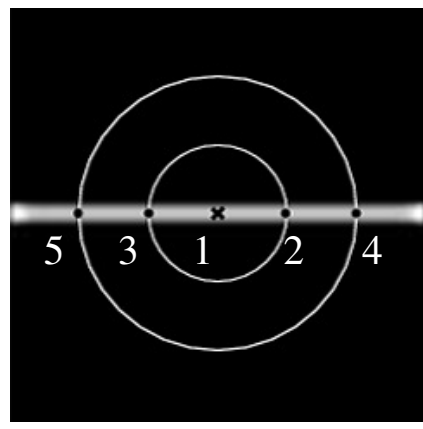

(b)

Figure 1: (a) A synthetic vessel-like structure used to configure a $B$-COSFIRE filter. (b) The output of a center-on DoG filter to the image in (a). The cross marker is the center of support of the $B$-COSFIRE filter while the enumerated spots represent the positions at which the strongest $D o G$ responses are achieved.

responds to vessel endings. In general, the two COSFIRE filters that we use respond to bar structures and thus we call them bar-selective COSFIRE filters or $B$ COSFIRE for brevity.

The rest of the paper is organized as follows: in Section 2 we present the $B$-COSFIRE filters; in Section 3 , we present the experimental protocol and the results achieved on four public data sets of retinal fundus images, which we compare with state-of-the-art methods in Section 4. Finally, we draw conclusions in Section 5.

\section{PROPOSED METHOD}

\section{$2.1 \quad$ B-COSFIRE filter}

A $B$-COSFIRE filter is trainable, in that its selectivity is determined by an automatic configuration process that analyzes a given prototype pattern. Figure 1a shows a synthetic bar structure that we use as prototype. The configuration procedure involves the convolution of a center-on Difference-of-Gaussians (DoG) filter - with a given standard deviation $\sigma$ of the outer Gaussian function ${ }^{1}$ - and the synthetic bar pattern followed by the determination of local maximum DoG responses along a number of concentric circles, Figure $1 \mathrm{~b}$. For each local maximum point $i$ we extract the polar coordinates $\left(\rho_{i}, \phi_{i}\right)$ with respect to the center of the prototype pattern. This location is indicated by the ' $x$ ' marker in Figure $1 b$.

This configuration procedure results in a set $S=$ $\left\{\left(\sigma_{i}, \rho_{i}, \phi_{i}\right) \mid i=1, \ldots, n\right\}$ of 3 -tuples. Here we use one DoG filter and thus the $\sigma$ values of all tuples are the same. This design decision allows the use of multi-scale DoG filtering to a prototype pattern of an irregular shape. As an example, the filter model automatically determined from the prototype pattern in

${ }^{1}$ The standard deviation of the inner Gaussian function is half the standard deviation of the outer Gaussian function.

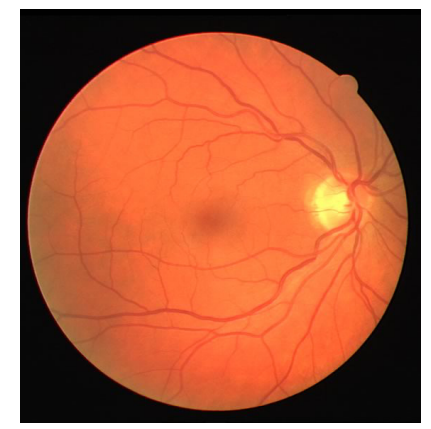

(a)

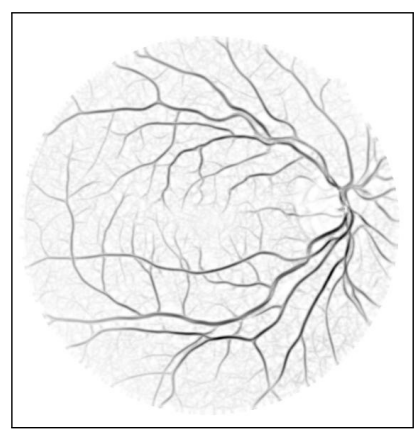

(b)
Figure 2: (a) Input retinal image and (b) the (inverted) output of a rotation-tolerant $B$-COSFIRE operator using 12 orientation preferences.

Figure $1 b$ is:

$$
S=\left\{\begin{array}{l}
\left(\sigma_{1}=2.6, \rho_{1}=0, \phi_{1}=0\right), \\
\left(\sigma_{2}=2.6, \rho_{2}=2, \phi_{2}=0\right), \\
\left(\sigma_{3}=2.6, \rho_{3}=2, \phi_{3}=3.14\right), \\
\left(\sigma_{4}=2.6, \rho_{4}=4, \phi_{4}=0\right), \\
\left(\sigma_{5}=2.6, \rho_{5}=4, \phi_{5}=3.14\right)
\end{array}\right\}
$$

We compute the response of a $B$-COSFIRE filter as the geometric mean of the responses of the concerned $D o G$ filters at the positions determined in the configuration step:

$r_{S}(x, y) \stackrel{\text { def }}{=}\left(\prod_{i=1}^{|S|}\left(s_{\sigma_{i}, \rho_{i}, \phi_{i}}(x, y)\right)\right)^{1 /|S|}$

where $s_{\sigma_{i}, \rho_{i}, \phi_{i}}(x, y)$ is the blurred response of a DoG filter with $\sigma_{i}$. Blurring is achieved by taking the maximum DoG response in a local neighourhood weighted by a Gaussian function. The Gaussian function is centered around the polar coordinates $\left(\rho_{i}, \phi_{i}\right)$ with respect to the center of the support of the $B$-COSFIRE filter (i.e. the pixel under consideration). It has a standard deviation $\sigma_{i}^{\prime}$ that is a linear function of the distance $\rho_{i}: \sigma_{i}^{\prime}=\sigma_{0}^{\prime}+\alpha \rho_{i}$. Further details about the parameters $\sigma_{0}^{\prime}$ and $\alpha$ are provided in Section 3.3. The blurring step is important as it allows for some tolerance with respect to the preferred positions of the concerned DoG responses.

In Azzopardi et al. (2014), we also demonstrate that the $A N D$-type function (geometric mean) that we use is more robust to noise than an $O R$-type function (e.g. arithmetic mean).

\subsection{Tolerance to rotations}

The $B$-COSFIRE filter configured above is selective to horizontal vessels. By using a rotated prototype pattern we can configure a filter that has preference to a different orientation. Alternatively, we create a new set $R_{\psi}(S)=\left\{\left(\sigma_{i}, \rho_{i}, \phi_{i}+\psi\right) \mid i=1, \ldots, n\right\}$ that represents a new $B$-COSFIRE filter with an orientation preference that is offset by $\psi$ radians from that of the 

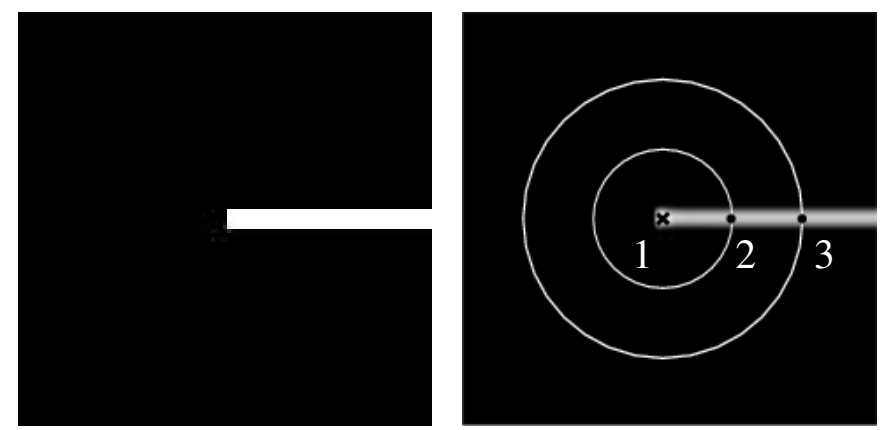

(a)

(b)

Figure 3: (b) Example of the configuration of an asymmetric $B$-COSFIRE filter by a prototype vesselending (a). The point of interest of the $B$-COSFIRE filter is indicated by the cross marker and lies on the end of the prototype.

original filter $S$. We denote by $\hat{r}_{S}(x, y)$ the rotationtolerant response at location $(x, y)$ that is achieved by taking the maximum value of various $B$-COSFIRE filters with different orientation preferences:

$\hat{r}_{S}(x, y) \stackrel{\text { def }}{=} \max _{\psi \in \Psi}\left\{r_{R_{\psi}(S)}(x, y)\right\}$

where $\Psi=\left\{0, \frac{\pi}{12}, \frac{\pi}{6}, \ldots, \frac{11 \pi}{12}\right\}$. Figure $2 b$ shows the rotation-tolerant output of a $B$-COSFIRE filter when applied to the green channel of the image in Figure 2a.

\subsection{Delineation algorithm}

The delineation algorithm that we propose uses two types of $B$-COSFIRE filters that we refer to them as symmetric and asymmetric. A symmetric $B$-COSFIRE filter is of the type configured above which considers the same number of DoG responses on both sides of the support center of the filter. Such a filter gives strong responses along vessels but responds weakly on the vessel endings. An asymmetric $B$-COSFIRE filter is one which achieves strong responses on vessel endings and it is configured by the prototype pattern shown in Figure 3a. Delineation is then achieved by thresholding the sum of the response maps of the symmetric and asymmetric $B$-COSFIRE filters. In Figure 4, we show examples of the output of the delineation algorithm (third column) and its thresholded version (fourth column) for each of the four data sets, together with the manually segmented ground truth (second column).

\section{EXPERIMENTS AND RESULTS}

\subsection{Data sets}

We use four data sets to evaluate the proposed approach: DRIVE (Staal et al. 2004), STARE (Hoover et al. 2000), CHASE_DB1 (Owen et al. 2009) and HRF (Odstrcilik et al. 2013).
The DRIVE data set is composed of 40 images (20 for training and 20 for testing) with resolution $768 \times 584$ pixels. For each image, a mask of the field of view (FOV) of the retina is provided together with the binary manual segmentation of the blood vessel tree. The images in the training set have been manually segmented by a single observer, while the images in the test set have been segmented by two other observers.

The STARE data set consists of 20 images $(700 \times$ 605 pixels), 10 of which contain signs of pathologies. Each image is distributed together with two manually segmented images provided by two different observers.

The CHASE_DB1 data set contains 28 colour images $(999 \times 960$ pixels $)$ from 14 patients in the program Child Heart And Health Study in England.The data set contains two groups of manually segmented images provided by two observers. For the three data set the first set of segmented images is used as ground truth.

The HRF data set consists of images with a resolution $(3504 \times 2336$ pixels $)$ that is substantially higher than images of the former data sets. It comprises of three sets of 15 images each collected from healthy people, patients with signs of glaucoma and patients with signs of diabetic retinopathy. Each image is coupled with one manually segmented image used as ground truth.

\subsection{Pre-processing}

For our experiments we consider only the green channel of the retinal fundus images as it provides the best contrast between the vessels and the background. Furthermore, we apply the pre-processing algorithm proposed by Soares et al. (2006) to smoothen the pixels that are close to the circumference of the FOV. This is achieved in an iterative way by increasing the radius of the FOV by one pixel in each iteration. The value of a new pixel is the average of the neighbouring pixels within the FOV that is determined in the pevious iteration. The initial FOV masks for the images of the DRIVE and HRF data sets are provided with the ground truth data. For the other data sets this information is, however, missing and we obtain the initial FOV masks by thresholding ${ }^{2}$ the luminosity channel of the CIELab version of the original RGB image. For the HRF data set, we downsampled the original images by a factor of 2 .

\subsection{Selection of parameters}

The $B$-COSFIRE filters that we propose have four parameters, namely the standard deviation $\sigma$ of the outer Gaussian function of the afferent DoG filter, a set of $\rho$ values that are the radii of the concentric circles used

${ }^{2}$ The thresholds are 0.5 and 0.1 for the STARE and CHASE_DB1, respectively. 


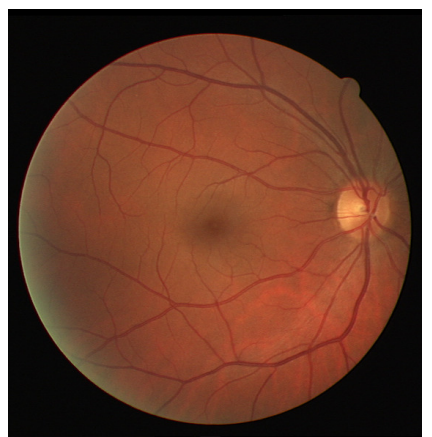

(a)

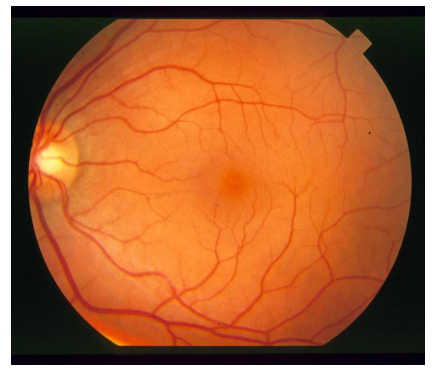

(e)

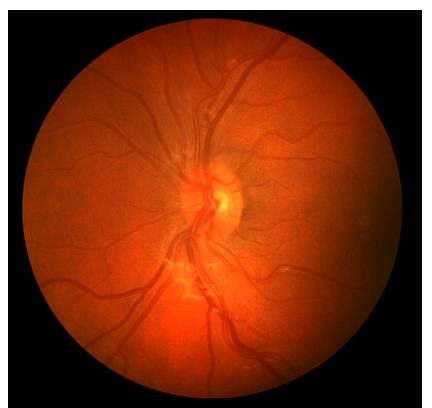

(i)

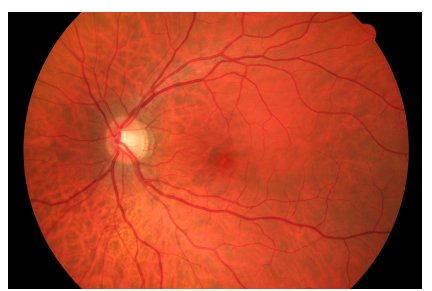

(m)

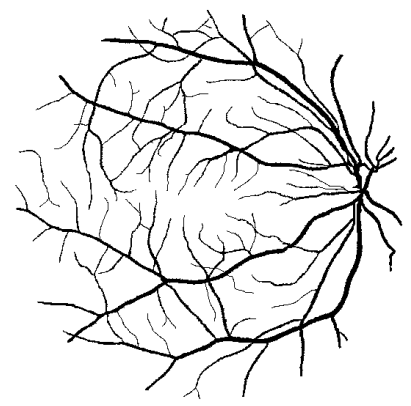

(b)

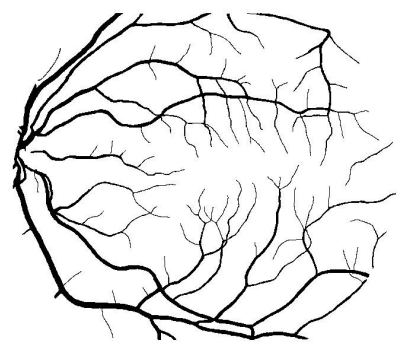

(f)

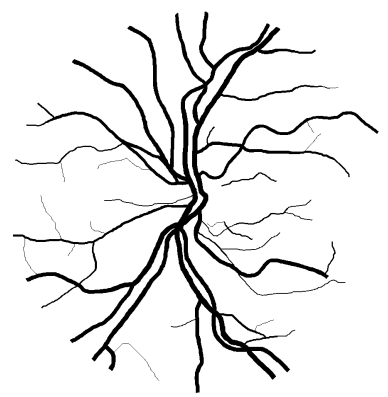

(j)

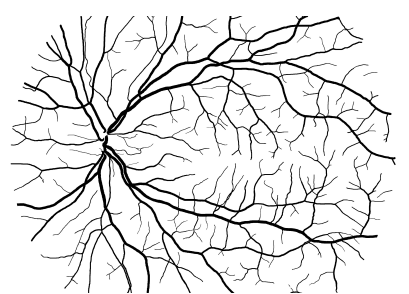

(n)

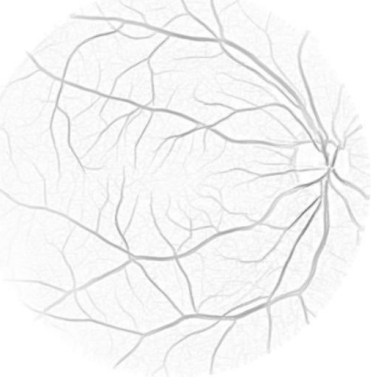

(c)

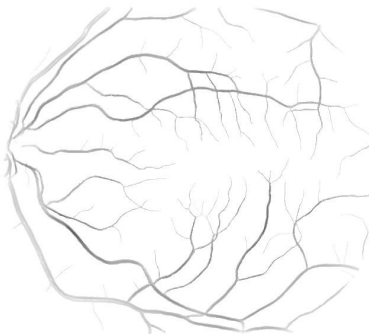

(g)

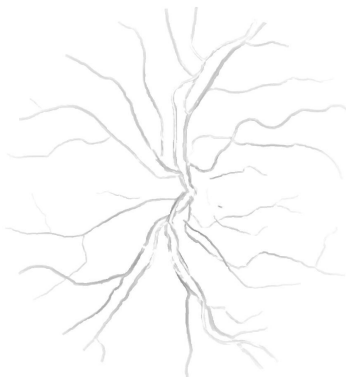

(k)

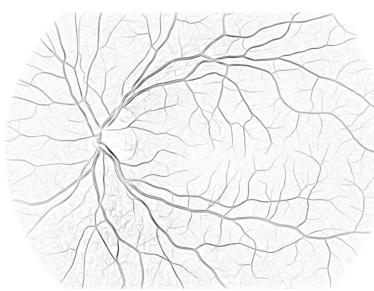

(o)

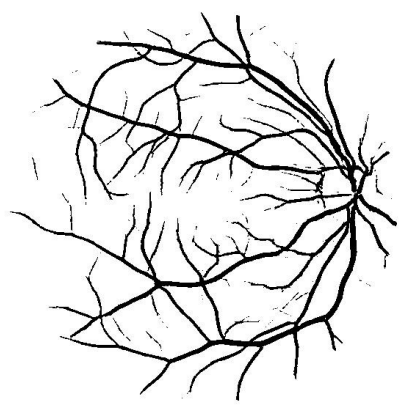

(d)

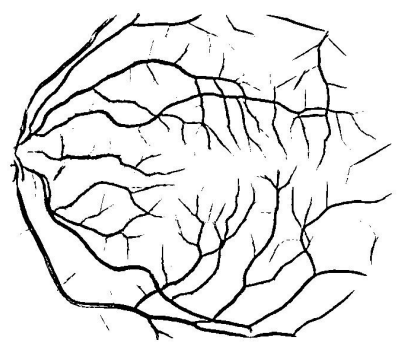

(h)

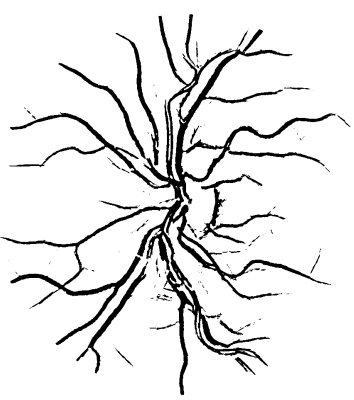

(1)

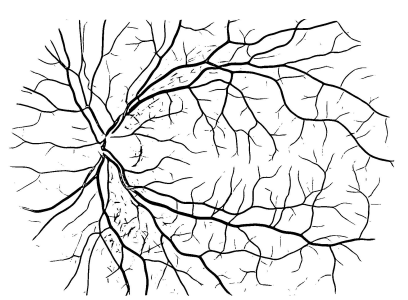

(p)

Figure 4: Examples of retinal fundus images (first column) from the four concerned data sets (DRIVE, STARE, CHASE_DB1 and HRF) together with the manually segmented ground truth (second column). The output of the delineation algorithm (third column) is thresholded in order to obtain the final segmented vessel tree (fourth column).

in the configuration, together with $\sigma_{0}^{\prime}$ and $\alpha$ that are used by the blurring function.

For each data set we consider a validation set of images and use a grid search to first estimate the parameter values of the symmetric $B$-COSFIRE filter and then to estimate the parameter values of the asymmetric $B$-COSFIRE filter that best improves on the performance of the symmetrical one. For DRIVE we use the provided 20 training images as the validation set, and for the other three data sets we form validation sets with the first half of the images.

In Table 1 we report the estimated parameter values of the two $B$-COSFIRE filters that we use to process the images in the four considered data sets.

\subsection{Evaluation method}

We binarize the summation of the response maps of the two $B$-COSFIRE filters by using a threshold value as a fraction of the maximum response. In this way, the pixels whose sum of the two $B$-COSFIRE filters is greater than the threshold are classified as vessels and the remaining as non-vessels.

We use a systematic set of threshold values between 0 and 1 in intervals of 0.01 and match every resulting binary image with the corresponding ground truth im- 


\begin{tabular}{l|l||c|c|c|c} 
Data set & Filter & $\sigma$ & $\rho$ & $\sigma_{\mathbf{0}}$ & $\alpha$ \\
\hline \hline \multirow{2}{*}{ DRIVE } & symmetric & 2.4 & $\{0,2, \ldots, 8\}$ & 3 & 0.7 \\
& asymmetric & 1.8 & $\{0,2, \ldots, 22\}$ & 2 & 0.1 \\
\hline \multirow{2}{*}{ STARE } & symmetric & 2.7 & $\{0,2, \ldots, 12\}$ & 1 & 0.6 \\
& asymmetric & 2.1 & $\{0,2, \ldots, 24\}$ & 1 & 0.1 \\
\hline \multirow{2}{*}{ CHASE_DB } & symmetric & 4.8 & $\{0,2, \ldots, 18\}$ & 3 & 0.2 \\
& asymmetric & 4.3 & $\{0,2, \ldots, 34\}$ & 1 & 0.1 \\
\hline \multirow{2}{*}{ HRF } & symmetric & 3.2 & $\{0,2, \ldots, 14\}$ & 3 & 0.3 \\
& asymmetric & 2.7 & $\{0,2, \ldots, 24\}$ & 1 & 0.1 \\
\hline
\end{tabular}

\begin{tabular}{l||c|c|c|c} 
Data set & Se & Sp & AUC & Acc \\
\hline DRIVE & 0.7655 & 0.9704 & 0.9614 & 0.9442 \\
STARE & 0.7763 & 0.9695 & 0.9555 & 0.9496 \\
CHASE_DB1 & 0.7699 & 0.9476 & 0.9497 & 0.9305 \\
HRF (Healthy) & 0.7467 & 0.9850 & 0.9557 & 0.9665 \\
HRF (DR) & 0.7620 & 0.9604 & 0.9413 & 0.9663 \\
HRF (Glaucoma) & 0.7446 & 0.9781 & 0.9561 & 0.9603 \\
\hline
\end{tabular}

Table 2: Experimental results of the proposed delineation algorithm on public data sets.

Table 1: Estimated parameter values of the $B$ COSFIRE filters used in four public data sets.

age. For each threshold value we compute the number of true positives, false positives, true negatives and false negatives, and subsequently use them to compute the Matthews Correlation Coefficient (MCC) as a performance indicator. The MCC is a suitable measurement for unbalanced classes and thus it is appropriate for the problem at hand where the background pixels outnumber the vessel pixels approximately by a factor of 7 .

Finally, we choose the threshold that provides the maximum average $\mathrm{MCC}$ value over a a validation data set, and report the Accuracy (Acc), Sensitivity (Se) and Specificity $(\mathrm{Sp})$ for that threshold. For the sake of completeness we also compute the ROC curve and its underlying area (AUC). In Table 2 we report the results that we achieve for the concerned four data sets.

\section{DISCUSSION}

In Table 4, we report the results achieved by the proposed method on the four considered data sets, in comparison with the ones achieved by other published methods. The results of these methods are achieved by thresholding the response of a filter or the score output of a classifier. However, the evaluation of algorithms for vessels segmentation in retinal fundus images is complex. The accuracy is not a suitable metric because it is biased by the high number of non-vessel pixels (background). Thus, in order to compare the proposed method with other published methods we move along the ROC curves. For the same specificity values reported in state-ofthe-art studies, we compare the value of the sensitivity. For the DRIVE data set and for the same specificity ( $S p=0.9764$ ) reported by (Mendonca and Campilho 2006) we achieve a sensitivity of 0.7376 , which is marginally better. Similarly, for the STARE data set and for the same specificity reported by (Mendonca and Campilho 2006) and (Al-Diri et al. 2009) ( $S p=0.9730$ and $S p=0.9681$ ) we achieve a sensitivity of 0.7554 and 0.7848 respectively, which is a significantly better result. We also achieve the best AUC value for the DRIVE data set with respect to all other unsupervised approaches $(A U C=0.9435$, AlRawi et al. $2007-A U C=0.9558$, Ricci and Perfetti $2007-A U C=0.9407$, Cinsdikici and Aydin $2009-$

\begin{tabular}{l||c}
\multicolumn{1}{c||}{ Method } & Processing time \\
\hline \hline B-COSFIRE & 10 seconds \\
(Jiang and Mojon 2003) & 20 seconds \\
(Staal et al. 2004) & 15 minutes \\
(Mendonca and Campilho 2006) & 2.5 minutes \\
(Soares et al. 2006) & 3 minutes \\
(Lam et al. 2010) & 13 minutes \\
(Marin et al. 2011) & 1.5 minutes \\
(Fraz et al. 2012) & 2 minutes \\
\hline
\end{tabular}

Table 3: Comparison of the time required to process an image from the DRIVE and STARE data sets.

\section{$A U C=0.9614$, Lam et al. 2010).}

For the HRF data set we achieve better results than the other published method (Odstrcilik et al. 2013). Indeed, for the same specificity achieved by Odstrcilik et al. 2013 for the healthy, diabetic retinopathy and glaucoma sets, we achieve sensitivity values of $0.8063,0.7528$ and 0.8168 , respectively. Such values are higher than the ones reported by Odstrcilik et al. 2013 (0.7861, 0.7463 and 0.79). As for the CHASE_DB 1 there are no other unsupervised methods to compare with. The only other state-of-the art method is a complex supervised approach (Fraz et al. 2012) based on an ensemble of classifiers.

As regards the time required to process an image (from DRIVE and STARE data sets), the proposed method is the most efficient with respect to other methods from the state of the art (Table 3 ).

\section{CONCLUSIONS}

The results that we achieve on DRIVE, STARE, CHASE_DB1 and HRF data sets demonstrate the high effectiveness of the proposed method. $B$-COSFIRE filters are versatile as they can be automatically configured to be selective for any given vessel-like pattern, including bifurcations and crossovers. We have made the Matlab implementation available online ${ }^{3}$. The processing of a $B$-COSFIRE filter is also very efficient. In fact, the proposed method is the most timeefficient algorithm for vessels delineation in retinal fundus images published so far.

\footnotetext{
${ }^{3}$ http://www.mathworks.com/matlabcentral/fileexchange/49172
} 


\begin{tabular}{|c|c|c|c|c|c|c|c|c|c|}
\hline & & \multicolumn{4}{|c|}{ DRIVE } & \multicolumn{4}{|c|}{ STARE } \\
\hline & Method & Se & Sp & AUC & Acc & Se & Sp & AUC & Acc \\
\hline \multirow{6}{*}{ 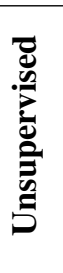 } & $B$-COSFIRE & 0.7655 & 0.9704 & 0.9614 & 0.9442 & 0.7716 & 0.9701 & 0.9563 & 0.9497 \\
\hline & (Mendonca and Campilho 2006) & 0.7344 & 0.9764 & - & 0.9463 & 0.6996 & 0.9730 & - & 0.9479 \\
\hline & (Martinez-Pérez et al. 2007) & 0.7246 & 0.9655 & - & 0.9344 & 0.7506 & 0.9569 & - & 0.9410 \\
\hline & (Al-Rawi et al. 2007) & - & - & 0.9435 & 0.9535 & - & - & 0.9467 & 0.9090 \\
\hline & (Ricci and Perfetti 2007) & - & - & 0.9558 & 0.9563 & - & - & 0.9602 & 0.9584 \\
\hline & (Lam et al. 2010) & - & - & 0.9614 & 0.9472 & - & - & 0.9739 & 0.9567 \\
\hline \multirow{6}{*}{ 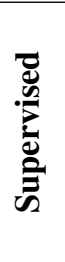 } & (Niemeijer et al. 2004) & - & - & 0.9294 & 0.9416 & - & - & - & - \\
\hline & (Staal et al. 2004) & - & - & 0.9520 & 0.9441 & - & - & 0.9614 & 0.9516 \\
\hline & (Soares et al. 2006) & 0.7332 & 0.9782 & 0.9614 & 0.9466 & 0.7207 & 0.9747 & 0.9671 & 0.9480 \\
\hline & (Ricci and Perfetti 2007) & - & - & 0.9633 & 0.9595 & - & - & 0.9680 & 0.9646 \\
\hline & (Marin et al. 2011) & 0.7067 & 0.9801 & 0.9588 & 0.9452 & 0.6944 & 0.9819 & 0.9769 & 0.9526 \\
\hline & (Fraz et al. 2012) & 0.7406 & 0.9807 & 0.9747 & 0.9480 & 0.7548 & 0.9763 & 0.9768 & 0.9534 \\
\hline
\end{tabular}

\begin{tabular}{|c|c|c|c|c|c|c|c|c|c|}
\hline & & \multicolumn{4}{|c|}{ CHASE_DB1 } & \multicolumn{4}{|c|}{ HRF } \\
\hline & Method & Se & $\mathbf{S p}$ & AUC & Acc & Se & Sp & AUC & Acc \\
\hline \multirow{2}{*}{ Unsup. } & B-COSFIRE & $\mathbf{0 . 7 5 8 5}$ & 0.9587 & 0.9487 & 0.9387 & 0.7511 & $\overline{0.9745}$ & $\overline{0.9510}$ & 0.9644 \\
\hline & (Odstrcilik et al. 2013) & - & - & - & - & 0.7740 & 0.9669 & 0.9493 & 0.9668 \\
\hline Sup. & (Fraz et al. 2012) & 0.7224 & 0.9711 & 0.9712 & $\overline{0.9469}$ & - & - & - & - \\
\hline
\end{tabular}

Table 4: Performance results of the proposed unsupervised $B$-COSFIRE filter approach on the concerned data sets compared to other methods from the state of the art.

\section{REFERENCES}

Al-Diri, B., A. Hunter, \& D. Steel (2009, Sept). An active contour model for segmenting and measuring retinal vessels. Medical Imaging, IEEE Transactions on 28(9), 1488-1497.

Al-Rawi, M., M. Qutaishat, \& M. Arrar (2007). An improved matched filter for blood vessel detection of digital retinal images. Computer in biology and medicine 37(2), 262-267.

Azzopardi, G. \& N. Petkov (2012). A CORF computational model of a simple cell that relies on LGN input outperforms the Gabor function model. Biol Cybern 106(3), 177-189.

Azzopardi, G. \& N. Petkov (2013). Trainable COSFIRE filters for keypoint detection and pattern recognition. IEEE Trans Pattern Anal Mach Intell 35, 490-503.

Azzopardi, G., A. Rodrguez-Snchez, J. Piater, \& N. Petkov (2014, 07). A push-pull corf model of a simple cell with antiphase inhibition improves snr and contour detection. PLoS ONE 9(7), e98424.

Azzopardi, G., N. Strisciuglio, M. Vento, \& N. Petkov (2014). Trainable cosfire filters for vessel delineation with application to retinal images. Medical Image Analysis 19(1), 4657.

Cinsdikici, M. G. \& D. Aydin (2009). Detection of blood vessels in ophthalmoscope images using mf/ant (matched filter/ant colony) algorithm. Computer Methods and Programs in Biomedicine, 85-95.

Fraz, M., P. Remagnino, A. Hoppe, B. Uyyanonvara, A. Rudnicka, C. Owen, \& S. Barman (2012). An ensemble classification-based approach applied to retinal blood vessel segmentation. IEEE Trans Biomed Eng 59(9), 2538-2548.

Hoover, A., V. Kouznetsova, \& M. Goldbaum (2000). Locating blood vessels in retinal images by piecewise threshold probing of a matched filter response. IEEE Trans Med Imaging 19(3), 203-210.

Jiang, X. \& D. Mojon (2003). Adaptive local thresholding by verification-based multithreshold probing with application to vessel detection in retinal images. IEEE Trans. Pattern Anal. Mach. Intell. 25(1), 131-137.

Lam, B., Y. Gao, \& A.-C. Liew (2010). General retinal vessel segmentation using regularization-based multiconcavity modeling. IEEE Trans on Med Imaging 29(7), 1369-1381.

Marin, D., A. Aquino, M. Emilio Gegundez-Arias, \&
J. Manuel Bravo (2011). A New Supervised Method for Blood Vessel Segmentation in Retinal Images by Using Gray-Level and Moment Invariants-Based Features. IEEE Trans Med Imaging 30(1), 146-158.

Martinez-Pérez, M. E., A. D. Hughes, S. A. Thom, A. A. Bharath, \& K. H. Parker (2007). Segmentation of blood vessels from red-free and fluorescein retinal images. Medical Image Analysis 11(1), 47-61.

Mendonca, A. M. \& A. Campilho (2006). Segmentation of retinal blood vessels by combining the detection of centerlines and morphological reconstruction. IEEE Trans Med Imaging 25(9), 1200-1213.

Niemeijer, M., J. Staal, B. van Ginneken, M. Loog, \& M. Abramoff (2004). Comparative study of retinal vessel segmentation methods on a new publicly available database. In Proc. of the SPIE - The International Society for Optical Engineering, pp. 648-56.

Odstrcilik, J., R. Kolar, A. Budai, J. Hornegger, J. Jan, J. Gazarek, T. Kubena, P. Cernosek, O. Svoboda, \& E. Angelopoulou (2013, Jun). Retinal vessel segmentation by improved matched filtering: evaluation on a new highresolution fundus image database. IET IMAGE PROCESSING 7(4), 373-383.

Owen, C. G., A. R. Rudnicka, R. Mullen, S. A. Barman, D. Monekosso, P. H. Whincup, J. Ng, \& C. Paterson (2009). Measuring retinal vessel tortuosity in 10 -year-old children: validation of the computer-assisted image analysis of the retina (caiar) program. Invest Ophthalmol Vis Sci 50(5), 2004-10.

Ricci, E. \& R. Perfetti (2007). Retinal blood vessel segmentation using line operators and support vector classification. IEEE Trans Med Imaging 26(10), 1357-1365.

Soares, J. V. B., J. J. G. Leandro, R. M. Cesar, Jr., H. F. Jelinek, \& M. J. Cree (2006). Retinal vessel segmentation using the 2-D Gabor wavelet and supervised classification. IEEE Trans Med Imaging 25(9), 1214-1222.

Staal, J., M. Abramoff, M. Niemeijer, M. Viergever, \& B. van Ginneken (2004). Ridge-based vessel segmentation in color images of the retina. IEEE Trans Med Imaging 23(4), 501509. 